\title{
docente para el \\ desarrollo de prácticas \\ pedagógicas con \\ medios informáticos y \\ telemáticos en el \\ contexto aula
}

Juny 2011. Pag. 59-79

ISSN 1135-1438

http://pedagogia.fcep.urv.cat/revistaut

\author{
Deysy Carolina Ramírez Conde ${ }^{a}$ y Ángel Pío González Sotob \\ Rebut: 25/04/2011 Acceptat: 02/06/2011
}

\begin{abstract}
Resumen
La Universidad de los Andes núcleo Dr. Pedro Rincón Gutiérrez, específicamente en el Programa de Profesionalización Docente (PPD), desarrolla cursos presenciales apoyados en entornos virtuales, concretamente la plataforma "MOODLE". Tomando como caso el curso Manejo de la plataforma Tecnológica Moodle (MPTM), curso en el cual los profesores han creado las aulas virtuales considerando la enseñanza de las herramientas meramente desde el aspecto técnico instrumental, dejando de lado el aspecto didáctico, surge la presente investigación. El estudio se ubica en la investigación cualitativa-cuantitativa y se desarrolló por fases: Preparatoria, trabajo de campo, analítica e interpretativa. Se aplicaron instrumentos de recolección de datos cualitativos: entrevista, autoinformes, diarios, y análisis de documentos. Así mismo, se implementaron instrumentos cuantitativos: cuestionarios en diversos momentos (antes, durante y después), para tener una aproximación a la realidad que circunscribe al objeto de estudio. Una vez analizados los datos se diseñó la propuesta de un modelo de acción docente basado en la actividad presencial apoyada en entornos virtuales y se aplicó en el rediseño del curso MPTM, el cual se validó y se concluyó que el modelo generado puede ser aplicado en cualquier asignatura que se desee desarrollar bajo la modalidad Semipresencial, ya que cubre las necesidades y requerimientos de los participantes del programa.
\end{abstract}

Palabras Clave: Medios Informáticos y Telemáticos, Plataforma MOODLE, BlendedLearning y Modelo Didáctico.

\footnotetext{
a Universidad de los Andes (ULA) Núcleo Dr. Pedro Rincón Gutiérrez, San Cristóbal Estada Táchira- Venezuela

b Universitat Rovira i Virgili . Tarragona
} 


\begin{abstract}
The Universidad de los Andes Dr. Pedro Rincón Gutiérrez core, specifically the Teacher Professional Development Program (PDP), develops classroom courses supported in virtual environments, specifically the platform "Moodle". Taking as a case management during the technological platform Moodle (MPTM) course in which teachers have created virtual classrooms considering teaching tools from the technical side merely instrumental, apart from the educational aspect, the present investigation. The studio is located in qualitative and quantitative research and developed in phases: Preparatory fieldwork, analytical and interpretive. Instruments were applied to collect qualitative data: interviews, self-reports, journals, and document analysis. Likewise, quantitative tools were implemented: questionnaires at various times (before, during and after) to have an approximation to reality that circumscribes the object of study. An analysis of the data was designed proposal for a model of action-based educational classroom activities supported in virtual environments and applied in the redesign of the course MPTM, which was validated and it was concluded that the generated model can be applied to any subject to be developed in a blended format, covering the needs and requirements of program participants.
\end{abstract}

Keywords: Media Informatics and Telematics, Plataform MOODLE, Blended-Learning and Teaching Model.

\title{
1. Introducción: Modelos didácticos en el desarrollo de prácticas pedagógicas con medios
}

Durante muchos años, se han desarrollado en el campo de la educación clases apoyadas en el modelo tradicionalista, siendo el rol del profesor instruir y aclarar dudas, logrando que el alumno participe a través de cuestionamientos dirigidos al grupo, tratando de llevar al estudiante hacia una participación inducida; de igual modo, asigna trabajos, tareas y proyectos para realizarse dentro y fuera de clase en forma individual o grupal, sin un proceso efectivo de tutoría.

Este modelo tradicionalista, por mucho tiempo, fue el que mejor se adaptaba a la disponibilidad de recursos y a las necesidades de la sociedad y de la comunidad académica. Sin embargo, los actuales cambios sociales y tecnológicos nos obligan a modificarlo, pero para modificarlo o introducir un modelo nuevo, hace falta realizar un estudio de la formación del docente de la actualidad, buscando el desarrollo de sus competencias, en función del perfil del futuro egresado, estimulando, a través de este nuevo modelo las mentalidades, valores y actitudes de los docentes hacia la tecnología, pero no sólo desde el punto de vista técnico sino también didáctico.

No cabe duda, que el profesorado no estará en condiciones de desarrollar prácticas pedagógicas de calidad con las tecnologías si sigue aplicando el modelo tradicional 
que fue realizado en los tiempos en que no existían los TICs emergentes para satisfacer las necesidades de formación.

Por esta razón, una meta educativa importante para las universidades es la formación de los profesores, como usuarios cualificados en el uso e implementación de las tecnologías y de la cultura que en torno a ellos se produce y difunde.

De allí que Padilla, A., Pedreros, A., Toledo, M. y Fuentes M. (2007), enuncian que:

"A los Profesores, acerca de su quehacer pedagógico, se les está demandando el buscar y crear nuevos escenarios de enseñanza que rompan los modelos bancarios y transmisivos de información, sobre contenidos o conocimiento que sólo se aprenden en el aula. Deben generar un espacio de discusión pedagógica que se caracterice por posicionamientos constructivos, participativos y colaborativos en torno a las TICs, que cambien el tradicional apoyo que poseen, fundamentalmente en los materiales impresos y en sus prácticas pedagógicas". (p. 2)

Es por ello que se requiere una profunda modificación de los planteamientos, modelos y métodos de enseñanza que hasta el día de hoy se han manejado.

Para Padilla et al. (2007), la problemática radica:

"Por una parte, al no existir una política clara de formación inicial y continua del profesorado en TICs, y por otra, de realizar esta, exclusivamente desde una óptica técnico-instrumental formándolos demasiado como usuarios de estos programas, pero poco o nada en lo pedagógico, para que aprendan incorporar esta herramienta a la práctica didáctica-curricular, y a crear entornos diferenciados de aprendizaje, constituyéndose esto en uno de los errores más significativos". (p. 5)

Todo ello nos conduce a que los Profesores deban adquirir un dominio no sólo basado en el manejo de la herramienta computacional (manejo técnico), "sino principalmente en lo que se refiere al conocimiento pedagógico de los contenidos que enseña y a la herramienta tecnológica como recurso de aprendizaje dentro del complejo acto didáctico, además de la disposición para el trabajo conjunto y el trabajo en red" (Padilla et al. 2007:6)

De lo anterior se puede decir que la tecnología por sí sola no asegura innovaciones, ni transformaciones en el quehacer educativo, si antes no se modifican los procedimientos pedagógicos con los cuales operan las TIC, dentro y fuera del aula de clase.

Es importante que el profesorado maneje la gran pregunta, ¿qué puede aportar esta nueva tecnología a mi actividad docente y cómo debo emplearla para mejorar mi actividad?, debido a que el campo didáctico, en el cual se aplica, presenta grandes cambios en la enseñanza superior, tal como lo enuncia Haug (2009:40): 
- Más atención al aprendizaje y menos a la enseñanza;

- Más atención al estudiante y menos poder al profesor;

- Prioridad a las exigencias de la sociedad frente a la independencia de la comunidad;

- Más atención al desarrollo de destrezas y habilidades, y menos a la mera adquisición de conocimientos

- Orientación al aprendizaje a lo largo de la vida, en vez de al enciclopedismo inicial, seguido de escasas posibilidades de formación posterior.

Siguiendo el mismo orden de ideas, queda claro que todos los cambios mencionados requieren un cambio en la forma de impartir las clases, pero para ello debe existir un conocimiento sobre los lineamientos al momento de ejercer la acción docente en el aula, a través de herramientas informáticas y telemáticas, evitando el uso meramente técnico. Para ello es necesario involucrar en gran medida el uso de las herramientas desde el punto de vista didáctico, de modo que el docente actual maneje una didáctica del uso e implementación de los medios informáticos y telemáticos bajo la teoría del constructivismo social, en el cual, se fomente el trabajo colaborativo, participativo, a través de entornos virtuales de formación, sobre la base de un modelo de acción docente en consonancia con la realidad.

De allí que sea necesario comenzar por preparar a los docentes en el uso didáctico de los medios, buscando el pleno desarrollo de competencias, cognitivas, metacognitivas y sociales, desde la acción docente como: comunicador, crítico, evaluativo, orientador, planificador, administrador e integrador.

La universidad de los Andes, concretamente el Núcleo Dr. Pedro Rincón Gutiérrez, no escapa de esta realidad y más aún en su Programa de Profesionalización Docente (PPD), el cual está dirigido a profesionales egresados de universidades e institutos de educación superior, con el título de Licenciados o equivalentes, cualquiera fuera su especialidad, son profesionales que ejercen la docencia sin poseer la titulación docente.

Este grupo de profesionales aspiran alcanzar la titulación docente en cinco semestres en cualquiera de las menciones del programa, el cual contempla cuatro áreas que conforman el plan de estudio: formación general, formación especializada, formación pedagógica y formación profesional, todas ellas conducentes a la formación pedagógica del docente. Estos futuros estudiantes del programa, ejercen la docencia en las diversas etapas y modalidades del sistema educativo venezolano, muchos de ellos implementan las TIC en el aula sin un uso adecuado de las mismas, sin tener un conocimiento didáctico, es decir de un modo meramente empírico basado en la experiencia.

Partiendo del supuesto anterior, se sugirió la creación de un curso basado en el manejo de la plataforma Moodle, desde el punto de vista del estudiante, el cual es 
actualmente, el curso de selección y sólo aquellos que lo aprueben podrán iniciar el Programa de Profesionalización Docente (PPD).

El objeto del Curso "Manejo de la plataforma Tecnológica Moodle" se centra en preparar al docente en formación para incursionar en una modalidad semipresencial, ya que es un profesional, que no dispone de tiempo, tiene formación en áreas ajenas al campo didáctico, con compromisos familiares, con horarios de trabajo establecidos, por lo que se requiere que el Programa de Profesionalización Docente (PPD) se desarrolle bajo la modalidad formativa flexible combinando la virtualidad con la presencialidad.

El problema central es que el curso fue diseñado sin tomar en cuenta las necesidades y requerimientos del estudiante, como profesional que ejerce la docencia, tampoco se consideraron los lineamientos del programa, ya que se presenta una aplicación meramente técnica, basado en el modelo tradicionalista, de ahí que se haga necesario analizar en detalle el curso, a fin de que el docente realice un proceso de formación de acuerdo a lo que plantea Aguilar (2008), para él, el docente debe estar:

"...más preparado para la búsqueda y utilización de metodologías más participativas, que derriben el concepto de un saber único, expositivo, de un modelo centrado en el profesor y en la enseñanza, que fue el eje de la educación tradicional; para dar paso a un nuevo enfoque que demanda una reorientación y una apertura hacia el auto aprendizaje y nuevas formas de enseñanza más activas y eficaces, estimulantes y potenciadoras de los procesos de construcción de conocimientos en los estudiantes" (p. 5)

El enfoque presentado anteriormente por Aguilar (2008) es esencial en la preparación del docente en formación que aspira formar parte del PPD, el cual es un sujeto que aprende, con conductas y constructos mentales desarrollados, con experiencias previas y estilos de aprendizaje propios, a la espera de una transformación en su forma de impartir clases e incluso innovar en el aula, a lo cual se le podría dar un inicio a través del curso Manejo de la Plataforma Moodle.

El problema no es introducir los medios informáticos y telemáticos en la educación en el proceso de enseñanza y aprendizaje, sino que se está repitiendo un modelo tradicional, el cual no es apto para esta nueva generación de estudiantes, ya no se trata de alfabetización tecnológica, ahora es formación a través de un modelo didáctico de acuerdo a las necesidades individuales e impredecibles del aprendizaje.

Lo que se persigue con este nuevo modelo didáctico es lograr un aprendizaje de alto nivel académico, relevante y actualizado, que fomente el desarrollo de la capacidad de autoaprendizaje, el aprendizaje colaborativo y la utilización de la plataforma tecnológica MOODLE del modo más adecuado y eficiente, en función del uso tanto técnico como didáctico desde el punto de vista del estudiante, con una visión de futuro para la implementación del mismo en su actuación docente. 


\section{Propósitos de la Investigación}

- Analizar teorías, modelos, planteamientos y estrategias involucradas en el desarrollo de prácticas pedagógicas con medios informáticos y telemáticos.

- Analizar en los profesores el dominio técnico- instrumental y didáctico de los medios informáticos y telemáticos en su práctica docente.

- Analizar la infraestructura en cuanto a medios informáticos y telemáticos con los que cuenta el profesorado en su ámbito laboral y de formación.

- Estudiar en los profesores el conocimiento, comprensión y aplicación de determinados criterios metodológicos para la selección, diseño, producción, utilización y evaluación de medios en situaciones de enseñanza-aprendizaje.

- Analizar en el Programa de Profesionalización Docente (PPD) los lineamientos sobre los cuales se afrontará la acción docente del profesional que imparte la docencia sin titulación.

- Establecer las bases para generar un Modelo de acción docente para el desarrollo de prácticas pedagógicas con medios informáticos y telemáticos en el aula.

- Adecuar el uso de las TIC en los procesos de enseñanza - aprendizaje del profesorado a través de un modelo educativo de acción docente.

- Facilitar el proceso de integración de las TIC como herramienta de trabajo e innovación docente en el PPD bajo la modalidad Blended- Learning.

- Lograr el desarrollo de competencias que les permitan a los profesores en formación aplicar los conocimientos adquiridos para resolver de forma significativa y coherente los problemas que se planteen en su ámbito educativo.

- Implementar el modelo educativo propuesto a través de un entorno virtual constructivista bajo la modalidad Blended Learning por medio de un curso virtual.

\section{Metodología de la investigación}

La investigación es cualitativa y cuantitativa, ya que el objeto de estudio está circunscrito al diseño de un curso en un entorno virtual de enseñanza / aprendizaje con modalidad Blended-Learning, específicamente para los estudiantes aspirantes a formar parte del Programa de Profesionalización Docente (PPD) de la Universidad de los Andes - Venezuela.

Se partió de una metodología descriptiva, asimismo la investigación se centra en el Estudio de Caso, específicamente en el curso de selección para formar parte del PPD, llamado "Manejo de la Plataforma Tecnológica Moodle" (MPTM), curso impartido en la Universidad de los Andes en el Núcleo Dr. Pedro Rincón Gutiérrez (Táchira- 
Venezuela). Este curso se dicta con la finalidad de preparar al profesional sin titulación docente en la utilización de los medios informáticos y telemáticos desde el punto de vista técnico- instrumental, teniendo claro la necesidad de cambiar el contenido y la forma de impartir el curso e incluso la necesidad de contar con un modelo de acción docente para impartir el curso no solo desde el punto de vista técnico- instrumental, sino también desde el punto de vista didáctico, surge la idea de que el curso se desarrolle dentro del modelo didáctico que se sugiere en la propuesta producto de la investigación.

La investigación se desarrolló en cuatro fases de acuerdo a lo enunciado por: Rodríguez, Gil y García (1999:64). (1) preparatoria, permitió diferenciar dos grandes etapas: Reflexiva y Diseño. La primera en la cual se estableció el marco teórico conceptual del cual partió la investigación y la segunda se centró en la planificación de actividades que se realizaron en las siguientes fases; (2) trabajo de campo, se centró en el acceso al campo, recogida productiva de datos y abandono del mismo, es decir, la interacción con los elementos en los cuales está circunscrito el objeto de estudio, fue la preparación de los instrumentos, el contacto con los sujetos; (3) analítica, se centró en la organización y distribución de los datos, así como el análisis de los mismos y en función de los resultados se elaboró la propuesta de la investigación; y (4) interpretativa, en esta última fase del proceso de investigación se presentó las conclusiones de la investigación y el informe final.

\subsection{Población y Muestra}

La población estuvo conformada por un total de 141 aspirantes a formar parte del Programa de Profesionalización Docente durante el periodo 2009-2010, los cuales fueron distribuidos en siete (07) secciones de trabajo, tal como se presenta en la tabla $\mathrm{N}^{\circ}$ 1:

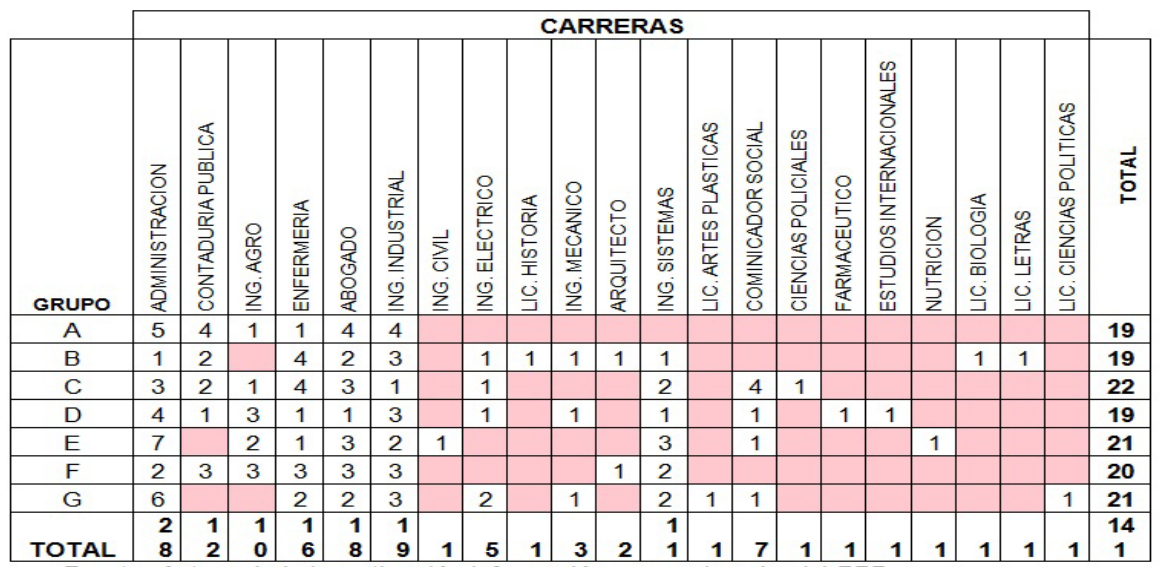

Tabla No 1: Estudiantes del PPD cohortes de la I a la IV.

Fuente: Autora de la investigación información proporcionada del PPD. 
La tabla anterior permitió tener una base de datos con respecto a la cantidad específica de acuerdo a la carrera de origen del participante, lo cual facilitó el proceso de selección de la muestra.

En lo que se refiere a las muestras de la investigación se trabajaron en total tres muestras: Muestra Inicial 1 (MI1), Muestra Inicial 2 (MI2) y Muestra Final (MF). Siendo muestras de tipo intencional y por criterios, siendo las dos últimas sub-muestras de la muestra Inicial I (MI1), tal como se evidencia en la figura $N^{\circ} 1$.

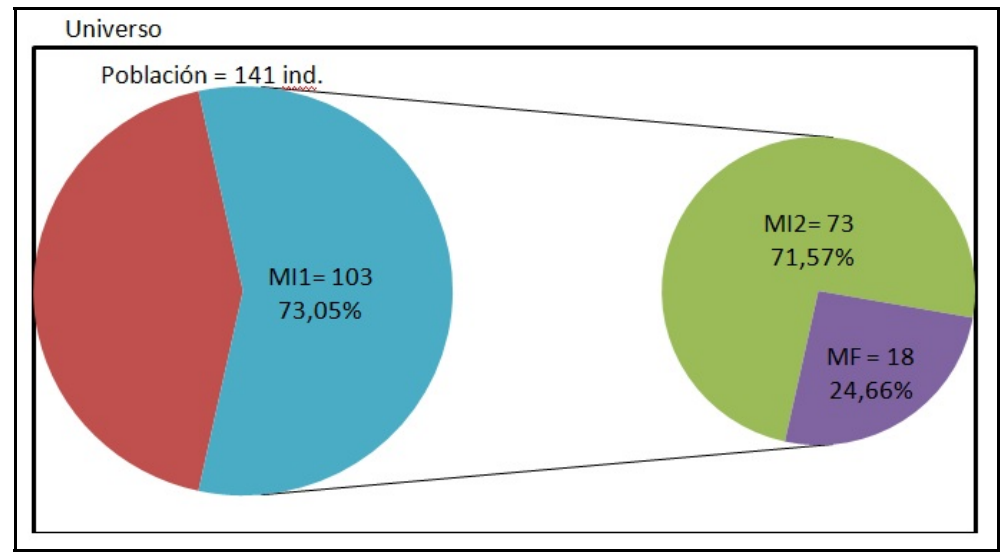

Figura $N^{\circ} 1$ : Muestras de la investigación

La MI1, se manejó específicamente en la fase trabajo de campo en la etapa detección de necesidades, la MI2 se trabajo en la fase analítica, es decir propiamente en la etapa validación del curso actual, así mismo la MF se manejó en la misma fase (Analítica), pero con el objeto de valorar lo aprendido en el curso de MOODLE en una de las asignaturas del Programa de Profesionalización Docente (PPD), específicamente en la asignatura "Didáctica de la Matemática", la cual se lleva a través de la plataforma MOODLE bajo la modalidad semipresencial (Blended Learning), a través de esta valoración se pudo determinar los cambios y mejoras con respecto al curso actual (Manejo de la plataforma Tecnológica MOODLE) a ser reflejadas en la propuesta de la investigación "Modelo Educativo de Acción Docente para el Desarrollo de Prácticas Pedagógicas Con Medios Informáticos y Telemáticos en el Aula".

\subsection{Instrumentos de recolección de datos}

La investigación congrega técnicas cuantitativas y cualitativas para recabar información. Con la combinación de esta estructura metodológica se realizó la triangulación de los datos y por tanto su validación. 
Las técnicas usadas para la recogida de datos fueron: El cuestionario, a fin de aplicarlo a: (a) Estudiantes aspirantes a cursar el curso de selección para formar parte del PPD; (b) Estudiantes que están actualmente cursando el curso y; (c) Estudiantes que ya están dentro del programa y que cursan asignaturas apoyadas con la plataforma MOODLE. Siendo en total tres (03) cuestionarios diferentes, los cuales se aplicaron según criterios establecidos por la investigadora, en tanto que es una técnica para constatar las necesidades, requerimientos, conocimientos, infraestructura y expectativas, de los aspirantes a formar parte del PPD de la ULA-Táchira, actuales docentes en las diversas etapas de la educación venezolana, con profesiones diversas, a fin de determinar las estrategias metodológicas, los recursos y las herramientas informáticas y telemáticas, el uso, aplicación en el aula, entre otros, de allí surgió la imperante necesidad de un modelo de acción docente, ya que actualmente se trabaja sin una estructura especifica.

También se establecieron técnicas cualitativas: (a) Entrevista individual, la cual se aplicó al profesor que imparte el curso de selección del PPD, referida a la forma en cómo se imparte el curso, estrategias, metodología, recursos, materiales y modelo didáctico sobre el cual se rige para el desarrollo del mismo, así como las inquietudes y necesidades por parte del profesorado; es decir, esta técnica permitió interpretar desde los propios agentes, las expectativas, demandas y análisis de los aspectos antes contemplados, generando una posición de necesidades de un modelo de acción para la implementación de medios informáticos y telemáticos; (b) análisis de documentos que conforman el desarrollo del curso, el programa, seguimiento de actividades en medios telemáticos, y el programa del PPD y; (c) autoinforme: Del profesor en este caso facilitador del curso y los diarios electrónicos de los participantes del curso a fin de conocer más a fondo su experiencia en el desarrollo del mismo.

Para alcanzar los objetivos planteados, se consideraron los niveles de análisis de datos manejados por Rodríguez, Gil y García (1999:313) tal como se contempla en la figura $\mathrm{N}^{\circ} 2$ : 


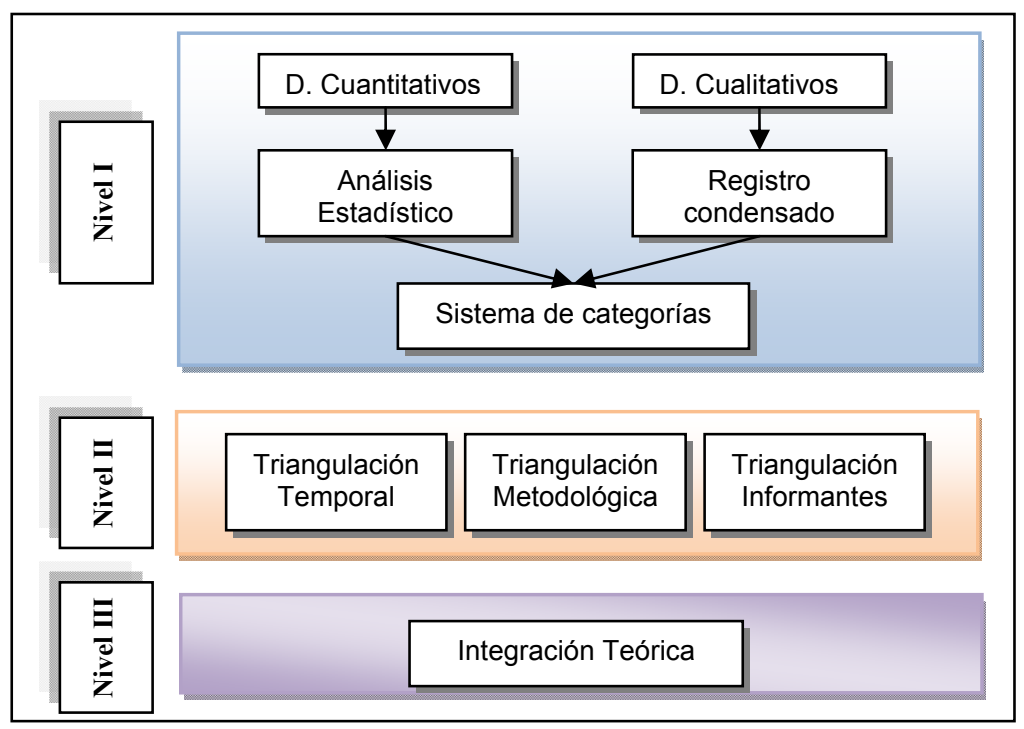

Figura $\mathrm{N}^{\circ}$ 2: Niveles de análisis de datos.

En el Nivel I, se realizó el análisis estadístico a través del paquete estadístico SPSS versión 17.0 y el análisis cualitativo por medio del programa Atlas ti. En lo que se refiere al Nivel II se llevó a cabo la triangulación de los datos de acuerdo a los elementos representados en la figura $\mathrm{N}^{\circ} 3$ :

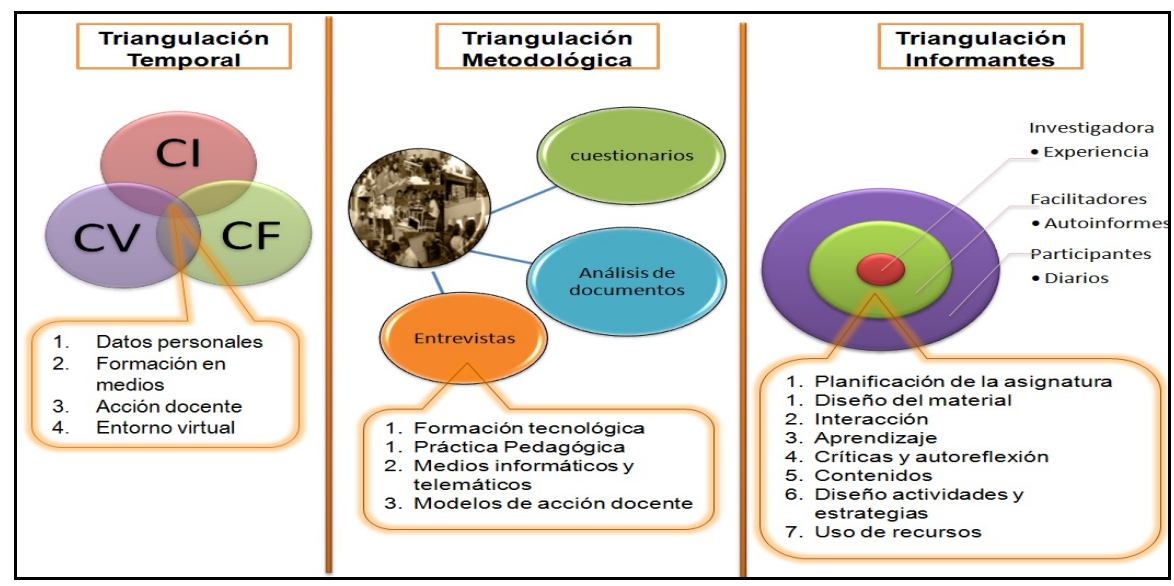

Figura Nº 3: Triangulación temporal, metodológica y de informantes de la investigación.

Así mismo, luego de triangular se manejó el Nivel III, en el cual se realizó la integración teórica que dio paso a los resultados de la investigación, a fin de tener 
bases para la propuesta del modelo y la implementación del mismo por medio del rediseño del curso objeto de estudio.

En lo referido a la triangulación temporal, se encontró en el ámbito formación en medios deficiencias desde el punto de vista de los contenidos, materiales del curso, metodología empleada, actividades propuestas y desarrolladas y los medios involucrados en el desarrollo del mismo; en el ámbito presencial el formador no cubrió en su totalidad las dudas de los participantes, no todas las orientaciones fueron recibidas en su oportuno momento, generando con ello vacíos en el proceso de formación, en lo referido a la virtualidad los participantes afirman que tanto los contenidos como las actividades desarrolladas en cada sesión virtual no fueron adecuados con el tiempo suministrado y faltó una planeación de un sistema tutorial tanto individual como grupal. Todo lo anterior deja claro en parte la necesidad de un subsistema de enseñanza que involucre el diseño de cursos en entornos virtuales del cual forme parte el facilitador y se involucre el aspecto de sistema tutorial, desarrollo de contenidos de acuerdo a las necesidades encontradas. De igual manera se consideró la opinión de los participantes en cuanto al entorno virtual, ya que se procuró conocer cómo les gustaría que fuera el curso, los elementos que debería manejar, entre otros aspectos de diseño, estructura y organización.

En la triangulación metodológica se cruzaron cuatro aspectos vitales para la determinación del modelo de acción docente, como fue la formación tecnológica, la práctica pedagógica, los medios informáticos y telemáticos y la presencia y aplicación de modelos didácticos. Todo lo anterior nos llevó a la conclusión de introducir un enfoque más pedagógico y menos instrumental, que los facilitadores tengan mayor asesoramiento en cuanto al diseño e implementación del curso, introducir nuevas formas de evaluación de acuerdo a los medios empleados, actualizar los contenidos del curso, introducir nuevas estrategias didácticas a fin de involucrar más el curso con el ámbito laboral de los participantes y de este modo hacer más funcional la práctica de las herramientas en casos de la vida real. Por ultimo existe una carencia por parte del facilitador en cuanto al elemento didáctico con los medios, incluso omiten el hecho de que el futuro docente debe ser capaz de implementar los medios sin mayor dificultad en su ámbito laboral, es evidente la necesidad de un modelo basado en principios, técnicas y el perfil del futuro egresado en cuanto al manejo didáctico de las TIC en su acción docente sin dejar de lado el aspecto de formación tecnológica en cuanto a manejo de herramientas, diseño de materiales y conocimiento, en conjunto con la práctica pedagógica adecuada que encierre lo concerniente a los objetivos, contenidos, metodología, recursos, materiales, evaluación y tutoría telemática, en base a un proceso de cambio e innovación de la mano con las herramientas informáticas y telemáticas en el contexto de aula.

Considerando los hallazgos encontrados en el análisis de los resultados, tomando tanto el punto de vista del facilitador, los participantes, los documentos de creación del Programa de Profesionalización Docente (PPD) y el propio PPD en sí, en pocas palabras partiendo de la triangulación temporal, metodológica y de informantes, surge la necesidad de realizar una propuesta sobre la base de un modelo Educativo 
de acción docente, generando lineamientos para la creación de cursos presenciales, apoyados en entornos virtuales de aprendizaje con una estructura técnica y didáctica, en la cual se logre satisfacer un conjunto de requerimientos, a continuación se mencionan los más relevantes para la investigación:

- Sobre la base de un modelo Educativo de acción docente generar lineamientos como propuesta para la generación de cursos virtuales con una estructura técnica y didáctica. Ya que dentro del análisis de los datos, lo que se refiere al modelo didáctico queda claro que confunden el modelo con las teorías de aprendizaje, muchos se refieren al constructivismo, mas no tienen claro un modelo didáctico, mucho menos uno en el cual se incluyan el manejo de las herramientas informáticas y telemáticas, es más solo identifican algunas estrategias didácticas manejadas en el curso actual, es decir es necesaria la actualización e implementación de estrategias acordes con la modalidad educativa en la que se va a incursionar.

- Actualizar recursos, materiales y herramientas en función a los objetivos del curso y los contenidos del mismo, de acuerdo a un subsistema de enseñanza en el cual el facilitador participe como diseñador del curso.

- Generar un proceso de evaluación adaptado a la autoevaluación, evaluación y co-evaluación fomentando el trabajo colaborativo, el trabajo en pequeños grupos, el trabajo individual, el estudio de casos, es decir diversas estrategias metodológicas y evaluativas aunado a un sistema tutorial propio de la actividad virtual dentro de una modalidad semi-presencial (Blended Learning).

Siguiendo el orden de ideas, en primera instancia, se tomaron los elementos necesarios para la generación de un modelo didáctico para el desarrollo de prácticas con herramientas informáticas y telemáticas, seguido del contraste de diversos modelos ya existentes a fin de generar un modelo propio de acuerdo a los requerimientos antes mencionados. En tal sentido se consideraron las siguientes teorías: Las dimensiones de un modelo planteadas por Salinas (2004), los componentes claves para el desarrollo de un modelo didáctico manejado por Socorro, Montenegro y Labidi, (2006), el Modelo didáctico mediador de Gimeno (1989), Modelos didácticos en entornos virtuales de formación de Salinas, J., Negre, F., Gallardo, A., Escandell, C. Y Torrandell, I (2005), elementos de un modelo didáctico de Salinas, J. et al. (2007), la triada de Henríquez, M.P. (2006), Elementos para la definición de un modelo pedagógico de Gairín, J. y Monereo, C. (2005), Un modelo pedagógico para la EaD de Pozzo, M. y Dobboletta, A. (2009), metodologías para cursos virtuales de SALINAS, J. (2005) y las fases para el Diseño de Cursos en Ambientes Virtuales de Sierra (2007). Una vez integradas las teorías de acuerdo a las necesidades y requerimientos detectados, se dio origen al modelo de acción docente propuesto producto de la investigación, tal como se evidencia en la figura $\mathrm{N}^{\circ} 4$ : 


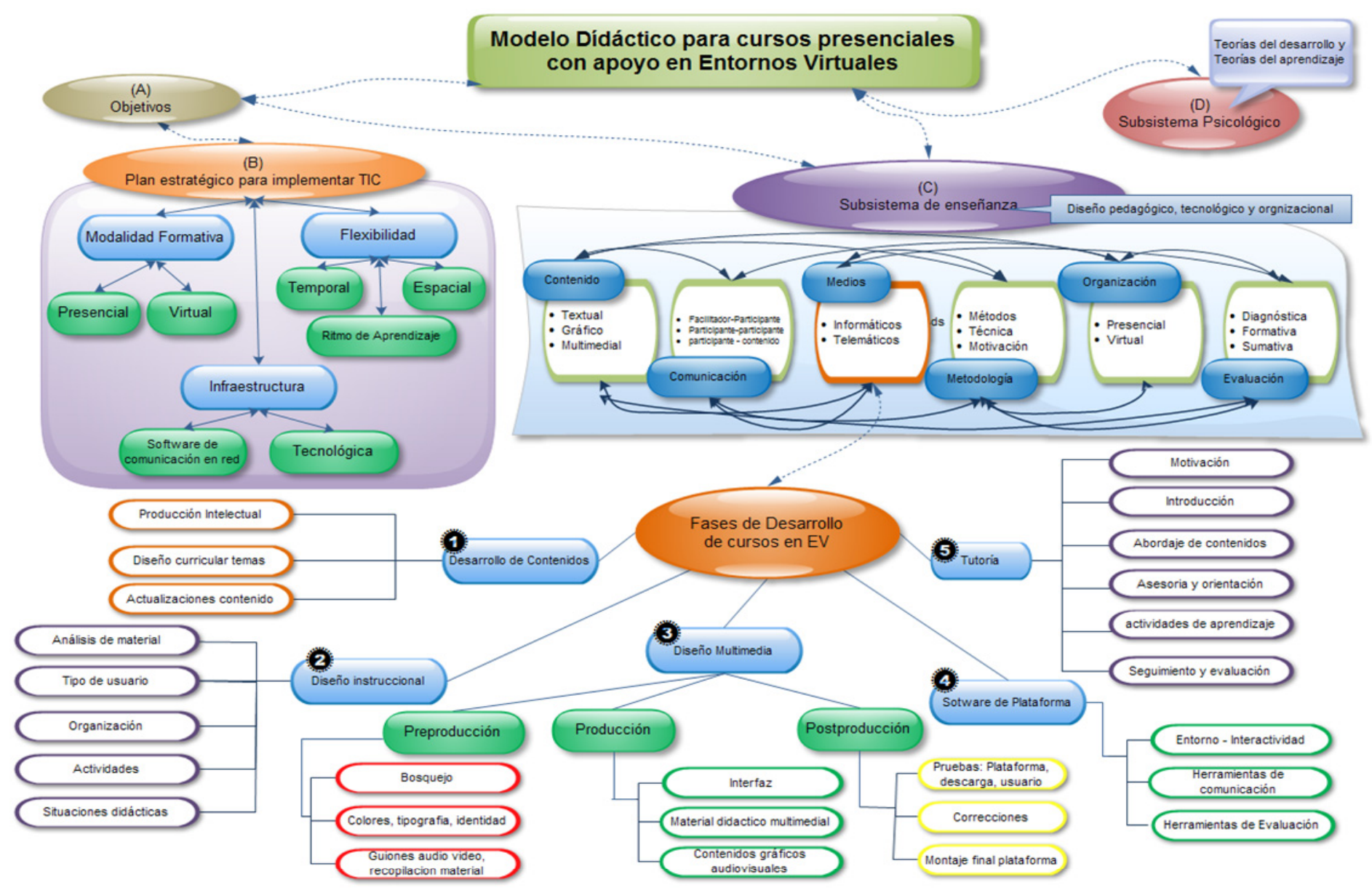

Figura No 4: Modelo Didáctico para el desarrollo de cursos presenciales con apoyo en Entornos virtuales (EV), bajo la modalidad BL en plataforma MOODLE. 
A continuación se detalla cada dimensión del modelo propuesto, el cual se estructura de la siguiente manera:

A. Los objetivos: Siendo un elemento clave para las subsiguientes dimensiones. Es el elemento clave para la toma de decisión en el ámbito didáctico, es decir en lo referido a la estrategia didáctica, las actividades a desarrollar, los resultados a obtener y como consecuencia un desarrollo más apropiado de la práctica didáctica, así como el trazado del plan estratégico de integración de las TIC. Es allí donde el facilitador debe trazar los objetivos del curso en función a las competencias de acuerdo al perfil del egresado.

B. Plan estratégico para implementar TIC: Esta segunda dimensión encierra la necesidad de trazar un plan referido a la modalidad formativa con la que se va a trabajar el curso, en este caso en concreto se manejó la modalidad Blended Learning (BL). Así mismo se debe considerar la infraestructura tanto el software de comunicación en red, en este caso se sugiere la plataforma MOODLE. Se debe determinar el nivel de flexibilidad en cuanto a tiempo, espacio y ritmo de aprendizaje, se le debe suministrar al usuario la posibilidad de mantener interacción con el curso desde casa, a cualquier hora y según un proceso de auto-aprendizaje orientado por el facilitador.

C. Subsistema de enseñanza: Comprende seis elementos claves dentro del sistema de enseñanza, los cuales mantienen una comunicación uno con todos, es decir cada uno precisa de la información y retroalimentación de los restantes, bajo un diseño pedagógico (objetivos, contenidos, metodología, materiales, actividades y evaluación), tecnológico (propiamente el diseño del curso en el entorno virtual, MOODLE) y organizacional (organización, identidad institucional y validación del curso).

D. Subsistema Psicológico: Este cuarto subsistema contempla las teorías de aprendizaje sobre las cuales se apoya el modelo propuesto, específicamente en el constructivismo social, en el cual el aprendiz aprende haciendo a través del trabajo colaborativo y/o cooperativo, en la medida en que interactúa con los medios, objetos, contenidos y sujetos; y (b) el aprendizaje significativo, para ello el aprendiz debe verse enfrentado a problemas reales que debe resolver, relacionándolo con sus conocimientos previos.

Una vez definido el modelo propuesto, se procedió a rediseñar el curso objeto de estudio con el fin de implementar el modelo y validarlo. Dando como resultado un curso virtual con objetivos bien definidos de acuerdo a las competencias del participante del PPD de consonancia al perfil de egresado de acuerdo a la carrera destino. De igual modo se trabajó el plan estratégico bajo la modalidad formativa Blended Learning $(\mathrm{BL})$, con el software de comunicación en red MOODLE.

El diseño del curso se desarrolló en el subsistema de enseñanza, comenzando por el diseño pedagógico en el cual se manejó el desarrollo del plan analítico, 
comprendiendo la selección de contenidos, medios, metodología, organización y evaluación. En el diseño tecnológico se manejó el diseño del curso por medio de la plataforma, seguido del montaje de los objetos de aprendizaje así como la selección y generación de herramientas de comunicación y colaboración, y por último los objetos de evaluación. Por último el diseño organizacional del curso quedo estructurado en cuatro unidades temáticas, con un apartado de cierre del curso y un apartado de mapa de navegación lineal del curso.

Cada unidad temática maneja la misma estructura y distribución de elementos de acuerdo a las necesidades y requerimientos del curso, es decir, se evidencia un botón para cada módulo dentro del curso, así como una relación imagen- tema, los módulos considerados fueron: Contenidos, vídeos, recursos, comunicación, prácticas, actividades, material adicional, evaluación y uso didáctico. Es necesario acotar que no todos los módulos fueron utilizados en todas las unidades temáticas, tal como se evidencia en la figura $\mathrm{N}^{\circ} 5, \mathrm{~N}^{\circ} 6$ y $\mathrm{N}^{\circ} 7$.

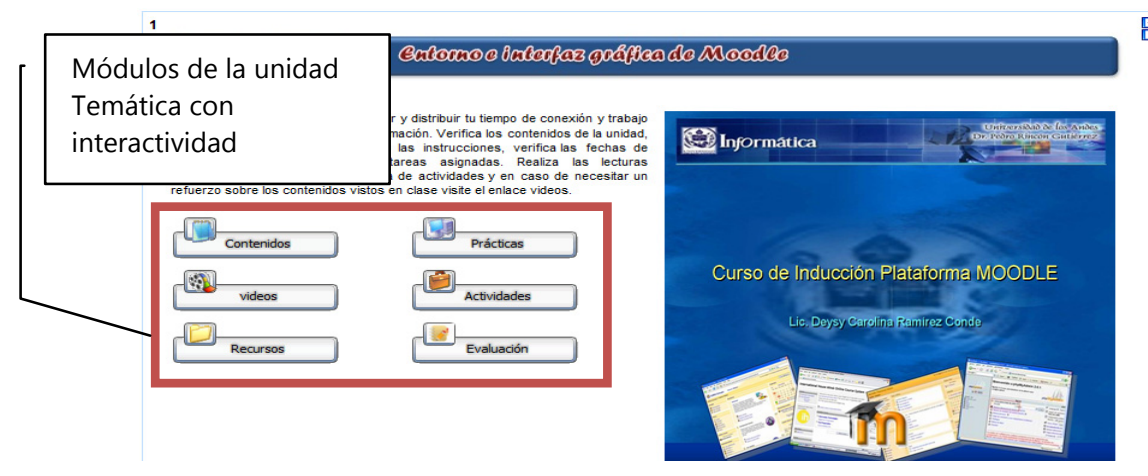

Figura N 5: Organización y presentación de los módulos de la unidad temática I

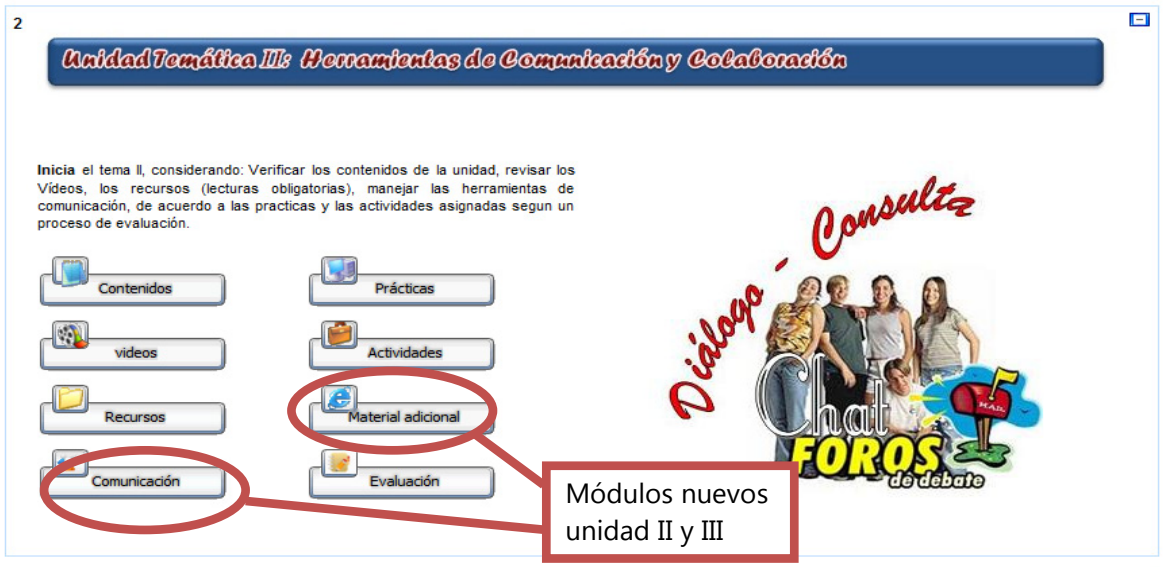

Figura Nº 6: Organización y módulos manejados en las unidades temáticas I y II 


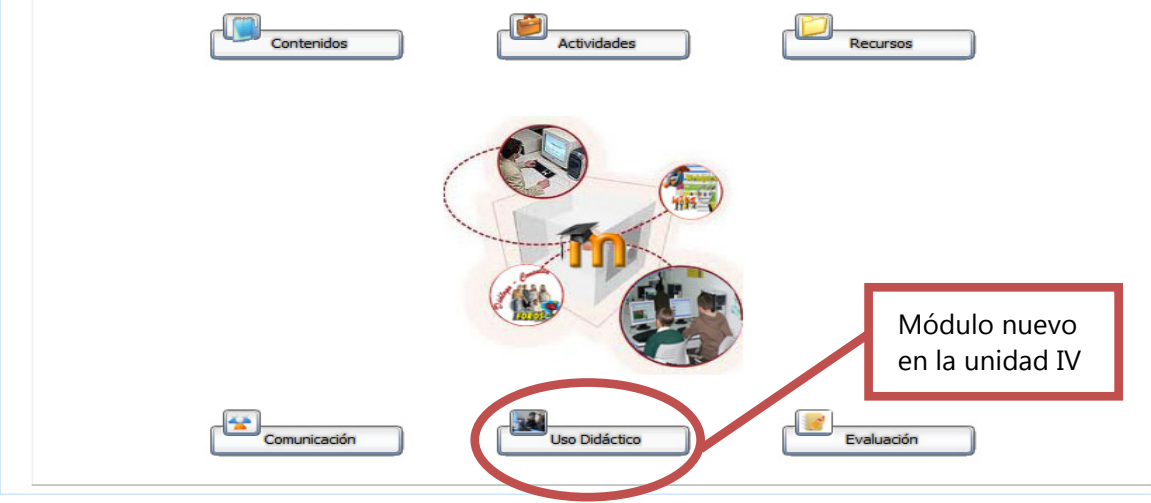

Figura $N^{\circ} 7$ : Estructura y organización de los módulos de la unidad temática IV

A continuación se detallan cada uno de los módulos:

Se trabajo la unidad temática $I$, centrada en los aspectos básicos de MOODLE, específicamente los conceptos, la interfaz gráfica del curso, la modificación del perfil, la elaboración del glosario

1. Contenidos: Presenta una página web con los contenidos de la unidad temática, para ubicar al participante en el tema, y que se forme una idea de los puntos que se van a tratar.

2. Vídeos: El módulo presenta una página web con enlaces a los vídeos sobre temas de difícil comprensión de acuerdo a las necesidades detectadas en cada unidad, los hipervínculos a cada vídeo lo traslada a otra web dentro del curso donde podrá visualizar el vídeo, con los controles del mismo y con navegación para volver a la página de los vídeos o ir a la página principal del curso. (Ver figura $\mathrm{N}^{\circ} 8$ )

3. Recursos: Dentro de los recursos se presenta una página web con las lecturas obligatorias de la unidad temática, los cuales se refieren a enlaces a documentos en formato .pdf, con la opción de descargar, guardar e imprimir.

4. Comunicación: El módulo de comunicación ofrece una web en la cual están los enlaces para establecer comunicación participante-participante, facilitador-participantes.

5. Prácticas: El módulo se refiere al conjunto de prácticas que se le ofrecen al participante como ejercitación de algunos de los contenidos de la unidad temática. 
6. Actividades: Se enlaza a una página web en la cual se estructura las actividades referidas al tema, tanto para la modalidad presencial como para la virtual, cada actividad virtual se enlaza a una web con los elementos didácticos necesarios para el desarrollo por parte del participante.

7. Material adicional: Es un módulo para ofrecer al participante material complementario basado en recursos de la web, con enlaces de tipo externo.

8. Evaluación: El módulo de evaluación ofrece la información sobre la actividad de evaluación, los criterios que serán considerados al momento de evaluar, la ponderación y el tipo de trabajo (individual o grupal).

9. Uso didáctico: El módulo de evaluación ofrece la información sobre la actividad de evaluación, los criterios que serán considerados al momento de evaluar, la ponderación y el tipo de trabajo (individual o grupal).

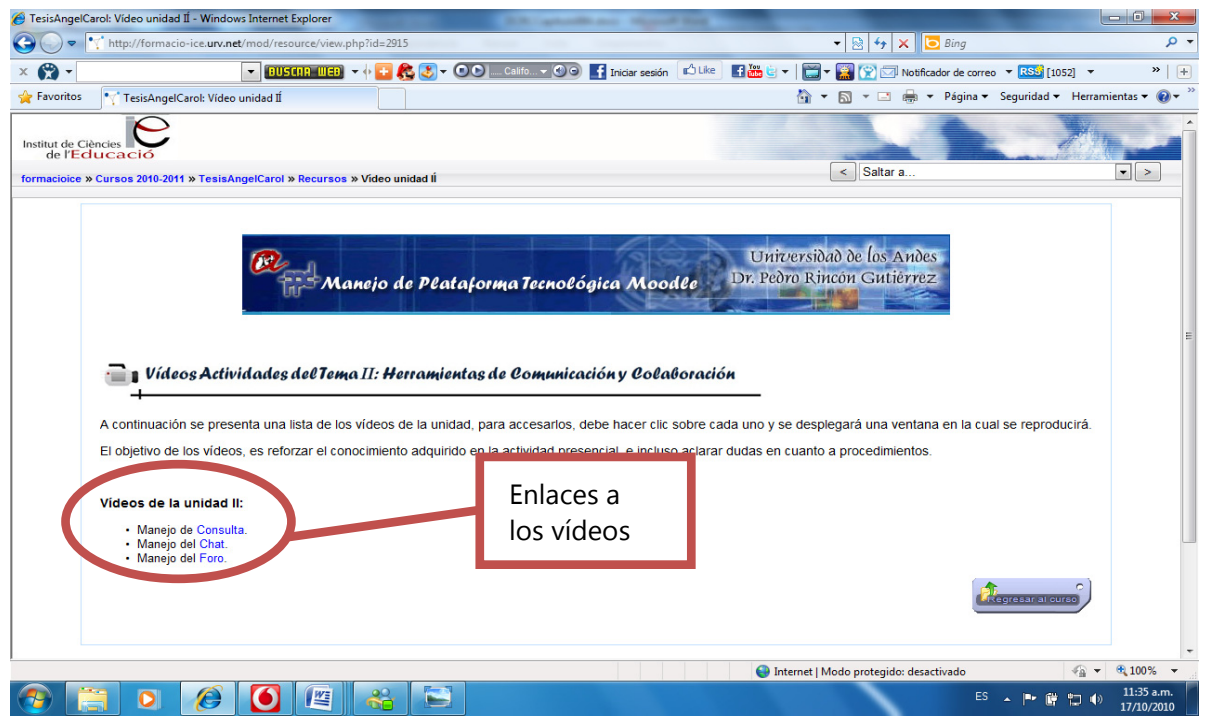

Figura Nº 8: Pantalla del módulo de vídeos

En la integración didáctica, se manejó como objeto de aprendizaje la WebQuest, Partiendo de la idea de suministrarle al estudiante materiales didácticos en formato web, en función de que genere proyectos de investigación sobre un tema o tópico, siguiendo una metodología de aprendizaje constructivista, se implementó la WebQuest a razón de integrar el uso didáctico de algunas de las herramientas que ofrece MOODLE, en una situación concreta de clase. 
Luego del diseño, se procedió a ejecutar la validación del curso la cual fue llevada a cabo por un grupo de expertos multidisciplinarios, a fin de obtener opiniones desde el punto de vista técnico, didáctico, del contenido y del manejo.

Se puede decir que fue plenamente satisfactorio el proceso de validación, ya que el $100 \%$ de los validadores en calidad de expertos, estuvo de acuerdo con la presencia de todas las dimensiones (diseño, organización, contenidos, interacción, comunicación y evaluación) en el curso, aparte de que se mostraron muy agradados e incluso impresionados por la forma como se manejo el curso. Lo cual deja entrever que efectivamente se cumplió con los requerimientos por parte del participante.

\section{Resultados de la investigación}

Cómo producto de la investigación se obtuvo lo siguiente:

- Un modelo de acción docente, aplicable a cualquier asignatura dentro del programa de Profesionalización Docente, e incluso extrapolable a otros contextos con características similares, ya sea para docentes en proceso de formación tanto inicial como para la formación continua. El modelo parte de los elementos claves para el desarrollo de un modelo didáctico, los cuales se adaptan al objeto de estudio, generando un alumno que se apropia del ambiente, interactúa, colabora, aprende, se comunica y se autoevalúa, con contenidos actualizados, apropiados a los objetivos de aprendizaje, los cuales están estructurados y organizados de modo que se le facilite al estudiante la comprensión de los mismos, también existe la meta de lograr el desarrollo del curso bajo la estructura de un modelo didáctico apoyado en entornos virtuales, con la ayuda de un método tanto para la actividad presencial como para la actividad virtual, donde el profesor diseña y maneja las etapas iniciales y de desarrollo de cursos virtuales bajo un modelo didáctico, manteniendo una relación alumno - profesor horizontal.

- Partiendo de la base del modelo didáctico, el mismo se implementó para generar el nuevo curso "Manejo de la Plataforma Tecnológica MOODLE", el cual ofrece una relación de los contenidos con los objetivos, un programa del curso, contempla las competencias del PPD, las funciones de facilitador, promotor social e investigador, así como las acciones docentes: comunicativa, autocritica y evaluativa, orientadora, planificadora, administradora e integradora.

- Una plantilla para el desarrollo de cursos virtuales en la plataforma MOODLE, con una estructura estándar, dinámica e interactiva, es decir, queda la base de una plantilla para que los profesores en este caso facilitadores que trabajen en el PPD, la implementen y solo quede es montar los contenidos de cada módulo. 
- Surge la necesidad de una segunda fase de la investigación donde los docentes en formación, manejen el curso desde el punto de vista de profesor, desarrollador de contenidos y gestor de las herramientas de comunicación, colaboración y evaluación que ofrece la plataforma MOODLE.

\section{A modo de Conclusión}

Los medios informáticos y telemáticos, concretamente a través de la plataforma MOODLE, ofrecen herramientas de comunicación, colaboración y evaluación adecuadas para la implementación de cursos bajo la modalidad semipresencial, en la cual es esencial la comunicación participantes-facilitadores y participantesparticipantes, al mismo tiempo deben existir actividades en función del desarrollo de competencias en las cuales se fomente el trabajo colaborativo así como el rol del docente tanto de facilitador, como de promotor social y de investigador, aunado a un sistema de evaluación de acuerdo a las necesidades formativas.

Esta nueva tecnología, no solo permite la implementación didáctica de los medios informáticos y telemáticos, sino también que el profesor se centre mas en el aprendizaje, es decir prestarle más atención al estudiante, en función de un estudiante más activo y participativo, de acuerdo a la adquisición de competencias especificas en cuanto a los diferentes modos de acción docente (comunicar, autocriticar, orientar, evaluar, integrar y gestionar).

En este mismo orden de ideas, se debe preparar al docente en formación, para resolver los problemas con los que se pueda encontrar tanto dentro como fuera del aula, en lo que incumbe a su práctica docente, pero siempre desde el punto de vista didáctico más que técnico instrumental, aunque está claro que los docentes que conforman los sujetos de la investigación, poseen la alfabetización en informática, pero carecen de una alfabetización del manejo técnico y didáctico de la plataforma MOODLE, por ello, es que se diseño y desarrolló el curso con el objeto de generar competencias en función de un uso didáctico adecuado de las herramientas que ofrece la plataforma.

\section{Referencias bibliográficas}

Aguilar, R. (2008). Las competencias básicas del Profesor Bimodal de la UTPL. Revista Cognición No 13 ISSN 1850-1974 Edición Especial II CONGRESO CREAD ANDES y II ENCUENTRO VIRTUAL EDUCA UTPLoja, Ecuador.

Gairín, J. y Monereo, C. (coord) (2005). Elementos para la definición de un modelo pedagógico de e-learning propio de la Universidad Autónoma de Barcelona (e-UAB). Universidad Autónoma de Barcelona. Documento electrónico [http://webs2002.uab.es/paplicada/htm/papers/e-learning_a_girona7.pdf] Visitado en (septiembre de 2009). 
Gimeno, J. (1989). Teoría de la enseñanza y desarrollo del curriculum. Madrid. Anaya.

Haug, G. (2009). El reto de la modernización universitaria en Europa en Gonzalez, A. y Fandos, M. (coord) (2009) La Internacionalització de les Universitats: El procés d'adaptació a l'espai europeu d'Educació Superior. Ediciones URV. Tarragona España. p. 37-47.

Henriquez, M.P. (2006). Diseño y evaluación de curso universitarios en modalidad híbrida: caso de la Asignatura Periodismo Digital en la universidad de los Andes. Universidad de los Andes Táchira. Venezuela.

Padilla, A, Pedreros, A., Toledo, M. Y Fuentes M. (2007). Las tics y los cambios en las prácticas pedagógicas: resultados de un pilotaje llevado a cabo en el marco del proyecto chile@prende1. Documento electrónico [http://www.redenlaces.cl/cedoc_publico/1226430628Art_culo_final_Chile_prende.pdf] visitado en sep. 2009

Perez, M y Aguaded, J (2004) Diseño de programas didácticos para integrar los medios y las tecnologías en el currículum escolar en Tecnologías para la educación, Diseño, producción y Evaluación de Medios para la formación docente. Editorial Alianza, Madrid, pp. 69-78, ISBN: 84-206-4188-X

Pozzo, M. y Dobboletta, A. (2009). Un modelo pedagógico para la EaD de enseñantes de E/LE. Universidad Nacional del Rosario. Argentina. Documento electrónico [www.salvador.edu.ar/vrid/publicaciones/Capacitacion/pozzo.doc] visitado en septiembre de 2009.

Rodriguez, Gil y Garcia (1999). Metodología de la investigación cualitativa. Segunda Edición. Málaga. España. Editorial Ajibe.

Salinas, J. (2004): Hacia un modelo de educación flexible: Elementos y reflexiones. En Martínez, F.; Prendes, MP. (coord.): Nuevas Tecnologías y Educación. Madrid: PearsonPrentice Hall. 145 -170.

Salinas, J. (2005). Propuesta de nuevas metodologías para el desarrollo de cursos de doctorado interuniversitarios mediante el aprendizaje electrónico. Informe final del proyecto EA2005-0215. Palma de Mallorca España.

Salinas, J., Negre, F., Gallardo, A., Escandell, C. y Torrandell, I (2005): Modelos didácticos en entornos virtuales de formación: identificación y valoración de elementos y relaciones en los diferentes niveles de gestión. EDUTEC '06. Congreso Internacional sobre La educación en entornos virtuales: calidad y efectividad en el eLearning. Tarragona (España)

Salinas, J., Negre, F., Gallardo, A., Escandell, C. y Torrandell, I (2007): Análisis de elementos que intervienen en el proceso de enseñanza aprendizaje en un entorno virtual de formación: Propuesta de un modelo didáctico. EDUTEC '07. Congreso Internacional sobre La educación en entornos virtuales: calidad y efectividad en el eLearning. Buenos Aires. Argentina. 
Sierra, H (2007). Modelo pedagógico humanista tecnológico de la dirección nacional de servicios académicos virtuales. Universidad Nacional de Colombia, Dirección Nacional de Servicios Académicos Virtuales. Bogotá - Colombia. Consulta electrónica [http://www.virtual.unal.edu.co/unvPortal/articles/ArticlesViewer.do?reqCode=viewDe tails\&idArticle=5\#section22] consultado (Mayo 2010).

Socorro, M., Montenegro, S. y Labidi, S. (2006). Un modelo didáctico- pedagógico mediador de aprendizaje cooperatica para educación a distancia en el CEFET-MA. International Conference on Multimedia and ICT in Education (ICTE). Lisboa- Portugal. Documento electrónico [www.formatex.org/micle2009] consultado en (Septiembre 2010)

\section{Nota biográfica}

Deysy Carolina Ramírez Conde, Profesora ordinaria de la Universidad de los Andes - Venezuela (ULA), a nivel de Asistente, Licenciada en Educación Mención Informática, Especialista en Informática Educativa, Magister en Educación Abierta y a Distancia, Doctora en Calidad y Reforma Educativa, Consejera del Dpto. de Computación e Informática. Representante profesoral del Programa de Doctorado en Pedagogía de la Universidad de los Andes. Ha sido Jefe (E) del Departamento de Computación e Informática de la ULA- Táchira. Forma parte del grupo de investigación FORTE-ICE. Forma Parte de la comisión de Acreditación y Equivalencias del Programa de Profesionalización Docente (PPD). Investigadora Activa. Email: carolinarc@ula.ve y ulaweb@hotmail.com.Teléfonos: 00584261792865 y 00582763424608.

Ángel-Pío González Soto, Catedrático de Universidad, del Área de Didáctica y Organización Escolar, adscrito al Departamento de Pedagogía de la Universidad "Rovira i Virgili". Doctor en pedagogía, director de centro de formación continua ICE de la URV. Experto en formación permanente en la empresa. Coordinador de los grupos de Investigación FORTE-ICE y CIFO. E-Mail: angelpio.gonzalez@urv.net y dirice@urv.net Teléfonos: 00.34.977.55.80.94 (Despacho Profesor). 00.34.977.55.80.66 (ICE). Fax: 00.34.977.55.80.75 y 00.34.977.55.80.73. 